\title{
RNP AND LEWIS RNP FOR ALL 14 NATURAL TENSOR NORMS OF GROTHENDIECK
}

\author{
S.M. MAEPA* \\ Department Mathematics and Applied Mathematics, University of Pretoria, \\ Pretoria 0002, South Africa. \\ E-Mail charles.maepa@up.ac.za
}

Abstract. We classify all 14 natural tensor norms of Grothendieck according to the possession or the lack of the Radon-Nikodym property (RNP) and the Lewis Radon-Nikodym property.

Mathematics Subject Classification (2000): 46B22, 46B28.

Key words: Tensor norm, Radon-Nikodym property, Lewis-Radon-Nikodym property.

1. Introduction. We will assume acquaintance with Grothendieck's Résumé [6] (cf. Diestel-Fourie-Swart [2] and Defant-Floret [1]).

A tensor norm (or $\otimes$-norm) $\alpha$ is said to have the Radon-Nikodym property $(\mathrm{RNP})$ if $\mathcal{L}^{\alpha}\left(X, Y^{*}\right)=X^{*} \stackrel{\alpha}{\otimes} Y^{*}$ isometrically for all Banach spaces $X$ and $Y^{*}$, where $Y^{*}$ has the approximation property and the Radon-Nikodym property (see Maepa [9, p. 234]). We say that $\alpha$ has the Lewis Radon-Nikodym property (Lewis $\mathrm{RNP}$ ) if $\mathcal{L}^{\alpha}\left(X, \ell^{1}\right)=X^{*} \stackrel{\alpha}{\otimes} \ell^{1}$ isometrically for every Banach space $X$ (see Lewis $[7$, p. 58]).

A $\otimes$-norm is said to be natural if it can be obtained from $\wedge$ by taking the dual, transpose, left or right injective or projective hull finitely often. The 14 natural $\otimes$-norms of Grothendieck appear in a table on page 163 of Diestel-Fourie-Swart [2] and each one in turn will be scrutinized for any possession of the RNP or the Lewis RNP or none of these.

Frequently useful in these deliberations are the following facts established in Maepa [9]:

Theorem 1. (Maepa [9, Theorem 3.1]) Let $\alpha$ be a $\otimes$-norm. If $\alpha$ has the Lewis $R N P$, then $\alpha /$ has the RNP.

Here $\alpha$ / is the tensor norm whose integral operators factor through an $L$-space $L$ into the bidual space with an $\alpha$-integral first factor and a bounded linear second factor.

*This work was completed while the author was on sabbatical at Kent State University (KSU), Kent, Ohio, USA in the Spring of 2010. Accordingly, the author wishes to thank the Department of Mathematical Sciences at KSU for providing resources and comfort to carry out the necessary academic tasks. Not the least in line to receive a heap of thanks is the Department of Mathematics and Applied Mathematics as well as the Office of the Dean: Natural and Agricultural Sciences, both of the author's home University of Pretoria, for supporting the visit financially. 
Theorem 2. (Maepa [9, Theorem 3.2]) If $\alpha$ has the RNP, then so does $\backslash \alpha$.

Here $\backslash \alpha$ is a tensor norm whose integral operators factor through a $C$-space $C$ into the bidual space with a bounded linear first factor and an $\alpha$-integral second factor.

Theorem 3. (Maepa [9, Theorem 3.3]) If $\alpha$ is symmetric and has the RNP, then so does $/ \alpha$.

Here $/ \alpha$ is a tensor norm such that for every $L$-space $L$ the composition of an $/ \alpha$-integral operator with every operator from $L$ is $\alpha$-integral.

Proposition 4. (Maepa [9, Proposition 3.4])

1. If $\alpha$ has the Lewis RNP, then so does $\backslash \alpha$.

2. If $\alpha$ is symmetric and has the Lewis RNP, then so does / $\alpha$.

2. The 14 natural $\otimes$-norms. The following result is basic to what is to follow:

Theorem 5. $\wedge$ has the RNP (and so has the Lewis RNP).

Proof. Let $X$ and $Y$ be Banach spaces such that $Y^{*}$ has the approximation property and the Radon-Nikodym property. By Diestel-Uhl Jr [3, Theorem VIII.4.6], which also appears implicitly in Grothendieck [5], $\mathcal{L}^{\wedge}\left(Y, X^{*}\right)=\mathcal{L}_{\wedge}\left(Y, X^{*}\right)$ isometrically. By Diestel-Uhl Jr [3, Corollary VIII.2.11], $\mathcal{L}^{\wedge}\left(Y, X^{*}\right)=\mathcal{L}^{\wedge}\left(X^{* *}, Y^{*}\right)$ isometrically. But $\mathcal{L}^{\wedge}\left(X, Y^{*}\right) \hookrightarrow \mathcal{L}^{\wedge}\left(X^{* *}, Y^{*}\right)$ is an isometric embedding, thanks to Diestel-Uhl Jr [3, Corollaries VIII.2.12 and 13]. Therefore

$$
\begin{aligned}
\mathcal{L}^{\wedge}\left(X, Y^{*}\right) & \hookrightarrow \mathcal{L}^{\wedge}\left(X^{* *}, Y^{*}\right) \\
& =\mathcal{L}^{\wedge}\left(Y, X^{*}\right) \\
& =\mathcal{L}_{\wedge}\left(Y, X^{*}\right) \\
& =X^{*} \hat{\otimes} Y^{*}
\end{aligned}
$$

since $Y^{*}$ has the approximation (cf. Diestel-Fourie-Swart [3, Proposition 1.5.5]) and $\wedge$ is symmetric. It follows that the embedding $\mathcal{L}^{\wedge}\left(X, Y^{*}\right) \hookrightarrow X^{*} \hat{\otimes} Y^{*}$ is isometric. Thus $X^{*} \hat{\otimes} Y^{*}=\mathcal{L}^{\wedge}\left(X, Y^{*}\right)$ isometrically by $Y^{*}$ 's approximation property, and so, the RNP of $\wedge$ has been confirmed.

Theorem 6. $\mathrm{C}^{*}=/ \wedge$ has the $R N P$.

Proof. This follows from Theorems 5 and 3 (in this order). 
Proof. This follows from Theorems 6 and 2 .

ThEOREM 8. $\mathrm{C}=\backslash \vee$ has the Lewis RNP.

Proof. For $u: X \longrightarrow \ell^{1}$ to be $\backslash \vee$-integral, $u$ must factorize through a $C$-space

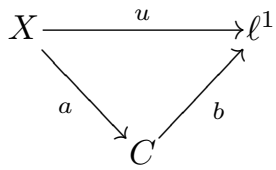

where $a$ and $b$ are bounded linear operators. Now, $b: C \longrightarrow \ell^{1}$ must be weakly compact because $\ell^{1}$ is weakly sequentially complete. By Schur's theorem $b$ is compact. So $b$ is the limit of a sequence $\left(b_{n}\right)$ of finite rank bounded linear operators $b_{n}: C \longrightarrow \ell^{1}$ - everything in sight has the metric approximation property - with $\left\|b_{n}\right\| \leq\|b\|$. It follows that $u$ is the $\backslash \vee$-integral-norm-limit of the sequence $u_{n}=b_{n} a$ of finite rank operators.

Theorem $9 . \mathrm{H}^{*}=\backslash \vee /$ has the $R N P$.

Proof. This follows from Theorem 1.

Note. For any tensor norm $\alpha$, all $\alpha$-nuclear operators are compact.

TheOREM 10. C fails the RNP.

Proof. Recall that an operator $u: X \longrightarrow Y$ is C-integral with $\|u\|_{\mathrm{C}} \leq 1$ if and only if there are a $C$-space $C$ and bounded linear operators $v: X \longrightarrow C$ and $w: C \longrightarrow Y^{* *}$, each of norm $\leq 1$, such that the following diagram commutes:

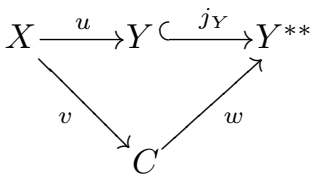

The C-integral norm of $u$ is $\|u\|_{\mathrm{C}}=\inf \{\|v\|\|w\|$ : the above factorization holds $\}$. Thus to show that $\mathrm{C}$ fails the RNP it suffices to find a C-integral operator that is not compact. Banach (cf. Grothendieck [4]) showed that there is an isometric quotient operator $q: C[0,1] \rightarrow \ell^{2}$ of $C[0,1]$ onto $\ell^{2}$; namely, the operator that takes $f \in C[0,1]$ to the sequence $\left(\int_{0}^{1} f(t) r_{n}(t) d t\right)_{n}$ where $\left(r_{n}\right)$ is the sequence of Rademacher functions. 
TheOREM 11. $\mathrm{H}=/ \wedge \backslash$ has the Lewis RNP, hence $\mathrm{H} /$ has the RNP.

Proof. That $\mathrm{H}$ has the Lewis RNP is a consequence of Proposition 5.6 of [9] and the consequence for $\mathrm{H} /$ is again an application of Theorem 1.

Recall that $u: X \longrightarrow Y$ is H-integral if $u$ admits a factorization

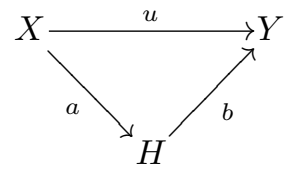

where $a, b$ are bounded linear operators; the H-integral norm of $u$ is $\|u\|_{\mathrm{H}}=$ $\inf \{\|a\|\|b\|$ : such that the above factorization holds $\}$.

TheOREM 12. H fails the RNP.

Proof. $\quad \mathrm{H}$ fails the RNP because $i d_{\ell^{2}}$ is H-integral but not compact.

THEOREM 13. $\backslash \mathrm{H}$ has the Lewis RNP but not the RNP.

Proof. This is a consequence of Theorem 11 and Proposition 4(1). Again, Banach's quotient operator $q: C[0,1] \rightarrow \ell^{2}$ is $\backslash \mathrm{H}$-integral but still is not compact.

THEOREM 14. $\gamma$ has the Lewis RNP but not the RNP.

Proof. If $u: X \longrightarrow \ell^{1}$ is $\gamma$-integral, then $u$ factorizes as follows:

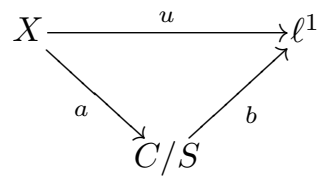

where $a, b$ are bounded linear operators and $C / S$ is a quotient of a $C$-space. Let $q: C \rightarrow C / S$ be the isometric quotient operator. Then $b\left(B_{C / S}\right)=b q\left(B_{C}\right)$. But $w q: C \longrightarrow \ell^{1}$ is compact, so $b q\left(B_{C}\right)$ is relatively compact, that is, $b\left(B_{C / S}\right)$ is relatively compact and $b: C / S \longrightarrow \ell^{1}$ is a compact operator. Again, $\ell^{1}$ has the metric approximation property, so there is a sequence $\left(b_{n}\right)$ of finite rank operators $b_{n}: C / S \longrightarrow \ell^{1}$ with $\left\|b_{n}\right\| \leq\|b\|$ so $b=$ operator $-\lim _{n} b_{n}$. If $u_{n}=b_{n} a$, then $u_{n}$ is a sequence of finite rank operators $u_{n}: X \longrightarrow \ell^{1}$ so that $\left\|u-u_{n}\right\|_{\gamma} \rightarrow 0$.

Now, $\gamma$ fails the RNP because the Banach quotient operator is $\gamma$-integral but not compact. 
Theorem 15. $\mathrm{L}=\mathrm{V} /$ fails the Lewis RNP.

Proof. $\quad$ Recall that $u: X \longrightarrow Y$ is L-integral if $u$ admits a factorization

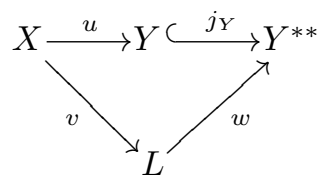

where $v, w$ are bounded linear operators; the L-integral norm of $u$ is $\|u\|_{\mathrm{L}}=$ $\inf \{\|v\|\|w\|$ : such that the above factorization holds $\}$. There is a quotient operator $q: L^{1}[0,1] \rightarrow \ell^{1}$ of $L^{1}[0,1]$ onto $\ell^{1} ; Q$ is L-integral but not compact.

Theorem 16. $\lambda=\mathrm{L} \backslash$ fails the Lewis RNP.

Proof. Recall that $u: X \longrightarrow Y$ is $\lambda$-integral with $\|u\|_{\lambda} \leq 1$ if and only if for every $C$-space $C$ and every operator $i: Y \longrightarrow C$ with $\|i\| \leq 1$, the operator $i u: X \longrightarrow C$ is L-integral with $\|i u\|_{\mathrm{L}} \leq 1$. The identity operator $i d_{\ell^{1}}$ on $\ell^{1}$ is $\lambda$-integral but not compact.

THEOREM 17. $\vee$ fails the Lewis RNP.

Proof. This is a consequence of Proposition 4.5 of [9].

TheOREM 18. $\mathrm{L}^{*}=\wedge \backslash$ fails the RNP.

Proof. After all, Grothendieck's Fundamental Inequality ensures that every operator from $\ell^{1}$ to $\ell^{2}$ is $\wedge \backslash$-integral; $\ell^{1} \hookrightarrow \ell^{2}$ is not compact and so is not $\wedge \backslash$ nuclear.

Open problem. We do not know if $\mathrm{L}^{*}=\wedge \backslash$ has the Lewis RNP.

TheOREM 19. $\lambda^{*}$ has the RNP if and only if it has the Lewis RNP.

Proof. We need only show that if $\lambda^{*}$ has the Lewis RNP, then it has the RNP. This in mind, suppose that $Y^{*}$ has the RNP and the approximation property and let $u: X \longrightarrow Y^{*}$ be $\lambda^{*}$-integral. Then $u$ factorizes as follows (cf. Diestel-Fourie-Swart [2, Corollary 2.4.14 (b)]):

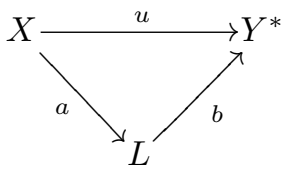


where $a$ is $\wedge \backslash$-integral (aka, absolutely summing), with $\|u\|_{\lambda^{*}}=\inf \left\{\|a\|_{\wedge} \backslash\|b\|\right.$ : such that the above factorization holds . But the Lewis-Stegall Theorem (DiestelUhl Jr [3, Theorem III.1.8] and Lewis-Stegall [8]) ensures that $b: L \longrightarrow Y^{*}$ factorizes as follows:

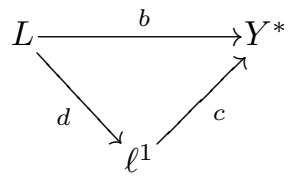

where $\|b\|=\inf \{\|c\|\|d\|$ : such that the above factorization holds $\}$. Of course, we now have the following pictorial description of $u$ :

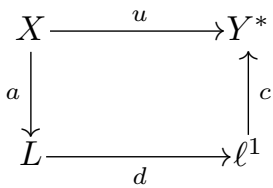

Since $\lambda^{*}$ has the Lewis RNP, $d a: X \longrightarrow \ell^{1}$ is $\lambda^{*}$-nuclear and so $c d a=u$ is too. The $\lambda^{*}$-nuclear norm of $u$ is easily seen to be $\leq\|a\|_{\wedge}\|d\|\|c\|$.

Next, we close with the

Open Problem. Does $\lambda^{*}$ have the Lewis RNP? Alternatively, is it so that if $u: X \longrightarrow L^{1}$ is absolutely summing and $v: L^{1} \longrightarrow \ell^{1}$ is a bounded linear operator, then $v u$ is quasi-nuclear?

For any normed spaces $X$ and $Y$, a bounded linear operator $u: X \longrightarrow Y$ is said to be quasi-nuclear if there exists a sequence $\left(x_{i}^{*}\right)$ in $X^{*}$ with $\sum_{i=1}^{\infty}\left\|x_{i}^{*}\right\|<\infty$ such that for every $x \in X$ it holds that $\|u x\| \leq \sum_{i=1}^{\infty}\left|<x, x_{i}^{*}>\right|$. Quasinuclear operators are treated in some detail in Pietsch ([10] and [11]). It is worth noting that $v u$ would be nuclear, and so quasi-nuclear (cf. [10, Satz 2.6] and [11, Proposition 3.2.5]), if either $v$ were also absolutely summing [11, Theorem 3.3.5] or there were a normed space $G$ containing $\ell^{1}$ such that $v u$ is nuclear as a mapping from $X$ into $G$ (cf. [10, Theorem 2.1] and [11, Proposition 3.2.7]).

\section{REFERENCES}

1. A. Defant and K. Floret, Tensor norms and operator ideals, North-Holland Mathematics Studies, Vol. 176, Elsevier Science Publishers B.V., Amsterdam, The Netherlands, 1993.

2. J. Diestel, J.H. Fourie And J. Swart, The metric theory of tensor products: Grothendieck's Résumé Revisited, American Mathematical Society, Providence, RI, 2008.

3. J. Diestel And J.J. Uhl JR, Vector measures, Mathematical Surveys, Vol. 15, American Mathematical Society, Providence, RI, 1977.

4. A. Grothendieck, Sur les applications linéaires faiblement compactes d'espaces du type $C(K)$, Canad. J. Math. 5 (1953), 129-173. 
5. Produits tensoriels topologiques et espaces nuléaires, Memoirs of the American Mathematical Society, Vol. 16, 1955.

6. —_ Résumé de la théorie métrique des produits tensoriels topologiques, Bol. Soc. Mat. São Paulo 8 (1956), 1-79.

7. D.R. LEwIS, Duals of tensor products, In: Banach spaces of analytical functions, (J. Baker, C. Cleaver and J. Diestel, eds.), Lecture Notes in Mathematics, Vol. 604, pp. 57-66, Springer-Verlag, Berlin/Heidelberg/New York, 1976.

8. D.R. Lewis AND C. Stegall, Banach spaces whose duals are isomorphic to $\ell_{1}(\Gamma)$, J. Funct. Analysis 12 (1973), 177-187.

9. S.M. MaePA, On the Radon-Nikodym property and the Lewis-Radon-Nikodym property for a tensor norm, Quaestiones Mathematicae 30 (2007), 231-245.

10. A. PIetsch, Quasinukleare Abbildungen in normierten Räumen, Math. Annalen 165 (1966), 76-90.

11. _ Nuclear locally convex spaces, Ergebnisse der Mathematik und ihrer Grenzgebiete, Band 66, Springer-Verlag, Berlin, 1972. 